\title{
HARDY AND RELLICH TYPE INEQUALITIES WITH TWO WEIGHT FUNCTIONS
}

\author{
Semra Ahmetolan And Ismail Kombe
}

Abstract. In the present paper we prove several sharp two-weight Hardy, Hardy-Poincaré, and Rellich type inequalities on the sub-Riemannian manifold $\mathbb{R}^{2 n+1}=\mathbb{R}^{n} \times \mathbb{R}^{n} \times \mathbb{R}$ defined by the vector fields:

$$
X_{j}=\frac{\partial}{\partial x_{j}}+2 k y_{j}|z|^{2 k-2} \frac{\partial}{\partial l}, \quad Y_{j}=\frac{\partial}{\partial y_{j}}-2 k x_{j}|z|^{2 k-2} \frac{\partial}{\partial l}, \quad j=1,2, \ldots, n
$$

where $(z, y)=(x, y, l) \in \mathbb{R}^{2 n+1},|z|=\left(|x|^{2}+|y|^{2}\right)^{1 / 2}$ and $k \geqslant 1$.

Mathematics subject classification (2010): 22E30, 26D10, 43A80.

Keywords and phrases: Hardy inequality with two weight functions, Rellich inequality with two weight functions, sharp constant.

\section{REFERENCES}

[1] B. Abdellaoui ANd I. Peral, Existence and nonexistence results for quasilinear eliptic equations involving the p-Laplacian with a critical potential, Annali di Matematica 182 (2003), 247-270.

[2] B. Abdellaoui, I. Peral And A. Prima, Strong regularizing effect of a gradient term in the heat equation with the Hardy potential, J. Func. Anal 258 (2010), 1247-1272

[3] S. Ahmetolan and S. Cavdar, On Nonlinear Degenerate Parabolic Equations with Time Dependent Singular Potential Functions, Mathematische Nachrichten 284, 10 (2011), 1219-1233.

[4] S. Ahmetolan And I. Kombe, A sharp uncertainty principle and Hardy-Poincaré inequalities on sub-Riemannian manifolds, Mathematical Inequalities \& Applications 15, 2 (2012), 457-467.

[5] G. J. Azorero And I. Peral, Hardy inequalities and some critical elliptic and parabolic problems, J. Diff. Equations 144 (1998), 441-476.

[6] P. Baras And J. A. Goldstein, The Heat Equation with a Singular Potential, Transactions of the American Mathematical Society 284, 1 (1984), 121-139.

[7] R. Beals, B. Gaveau And P. Greiner, On a Geometric Formula for the Fundamental Solution of Subelliptic Laplacians, Mathematische Nachrichten 181 (1996), 81-163.

[8] R. Beals, B. Gaveau and P. Greiner, Green's Functions for Some Highly Degenerate Elliptic Operators, Journal of Functional Analysis 165 (1999), 407-429.

[9] H. BREZIS AND J. L. VÁZQUEZ, Blow-up solutions of some nonlinear elliptic problems, Rev. Mat. de la Universidad Complutense de Madrid 10, 2 (1997), 443-469.

[10] X. CABRÉ AND Y. MARTEL, Existence versus explosion instantanée pour des équetions de la chaleur linéaires avec, potentiel singulier, C. R. Acad. Sci. Paris 329, 1 (1999), 973-978.

[11] G. B. Folland, Fundamental solution for a subelliptic operator, Bull. Amer. Math. Soc. 79, 2 (1973), 373-376.

[12] J. A. Goldstein And I. Kombe, Nonlinear Degenerate Parabolic Equations with Singular LowerOrder Term, Advances in Differential Equations 8, 10 (2003), 1153-1192.

[13] G. R. Goldstein, J. A. Goldstein And A. Rhandi, Weighted Hardy's inequality and the Kolmogorov equation perturbed by an inverse-square potential, Appl. Anal. 91, 11 (2012), 2057-2071.

[14] P. C. GREINER, A fundamental Solution for non-elliptic partial differential operator, Canadian Journal of Mathematices 31 (1979), 1107-1120.

[15] G. H. HARDY, Note on a theorem of Hilbert, Math. Zeitschr. 6 (1920), 314-317. 
[16] D. HAUER AND A. RHANDI, A weighted Hardy inequality and nonexistence of positive solutions, Arch. Math. (Basel) 100, 3 (2013), 273-287.

[17] L. Hörmander, Hypoelliptic Second Order Differential Equations, Acta Mathematica 119 (1967), $147-171$.

[18] I. KомвE, The Linear Heat Equation with Highly Oscillating Potential, Proceeding of the American Mathematical Society 132, 9 (2004), 2683-2691.

[19] I. Kомве, On the Nonexistence of Positive Solutions to Nonlinear Degenerate Parabolic Equations with Singular Coefficients, Applicable Analysis 85, 5 (2006), 467-478.

[20] I. KomBE, Sharp Weighted Rellich and Uncertainty Principle inequalities on Carnot Groups, Communications in Applied Analysis 14 (2010), no. 2, 251 14, 2 (2010), 251-272.

[21] A. Moradifam And N. Ghoussoub, Bessel pairs and optimal Hardy and Hardy-Rellich inequalities, Math. Ann. 349, 1 (2011), 1-57.

[22] P. NiU, Y. OU AND J. HAN, Several Hardy type inequalities with Weights related to generalized greiner operator, Canadian Math. Bulletin 53, 1 (2010), 153-162.

[23] P. NiU, H. Zhang And Y. WAng, Hardy Thype and Rellich Type Inequalities on the Heisenberg Group, Proceeding. Amer. Math. Soc. 129 (2001), 3623-3630.

[24] I. PERAL AND J. L. VÁZQUEZ, On the stability or insitability of the singular solution of the semilinear heat equation with exponential reaction term, Arch. Rational Mech. Anal. 129 (1995), 201-224.

[25] F. RELlich, Halbbeschränkte Differentialoperatoren häherer Ordnung, Proceedings of the International Congress of Mathematicians, Edit by J. C. H. Gerretsen et all, Amsterdam 3 (1956), 243-250.

[26] J. VAncostenoble And E. ZuAZuA, Hardy inequalities, observability, and control for the wave and Schöndirger equations with singular potentials, SIAM journal on Math. Anal. 41, 4 (2009), 15081532.

[27] J. L. VÁzQuez And E. ZuAZUA, The Hardy constant and the asymptotic behaviour of the heat equation with an inverse-square potential, J. Funct. Anal. 173 (2000), 103-153.

[28] H. ZHANG AND P. NiU, Hardy-type inequalities and Pohozaev-type identities for a class of pdegenerate subelliptic operators and applications, Nonlinear Anal. 54, 1 (2003), 165-186. 